\title{
The Current Prevalence of Irritable Bowel Syndrome in Asia
}

\author{
Full-Young Chang, MD*, Ching-Liang Lu, MD and Tseng-Shing Chen, MD \\ Division of Gastroenterology, Taipei Veterans General Hospital and National Yang-Ming University School of Medicine, Taipei, Taiwan
}

Irritable bowel syndrome (IBS) has been one of the commonly presented gastrointestinal disorders. It is of interest how commonly it presents in the society. Western studies indicated that most population-based IBS prevalences range $10 \%-15 \%$. It is believed that IBS is prevalent in both East and West countries without a significant prevalence difference. Most recently, the Asia IBS prevalence has a higher trend in the affluent cities compared to South Asia. Since many Asia IBS prevalence studies have been published in the recent decade, we could compare the IBS prevalence data divided by various criteria in looking whether they were also comparable to this of West community. Summarized together, most Asia community IBS prevalences based on various criteria are usually within the range 1\%-10\% and are apparently lower than these of selected populations. Within the same population, the prevalence orders are first higher based on Manning criteria, then followed by Rome I criteria and finally reported in Rome II criteria. Overall, the median value of Asia IBS prevalences defined by various criteria ranges $6.5 \%-10.1 \%$. With regard to gender difference, female predominance is usually found but not uniquely existed. For the IBS subtypes, the proportions of diarrhea predominant-IBS distribute widely from $0.8 \%$ to $74.0 \%$, while constipation predominant-IBS proportion ranges $12 \%-77 \%$. In conclusions, current Asia IBS prevalence is at least equal to the Western countries. Female predominant prevalence in Asia is common but not uniquely existed, while the proportions of IBS subtypes are too variable to find a rule.

(J Neurogastroenterol Motil 2010;16:389-400)

\section{Key Words}

Asia; Gastrointestinal motility; Irritable bowel syndrome; Prevalence

\section{Introduction}

Upon Rome III definition, irritable bowel syndrome (IBS) has been a biopsychosocial dysfunction. It means that a biological bowel dysfunction is the final result of brain-gut linkage and modified by the social, environmental and psychological factors. ${ }^{1,2}$ Since a well-known functional disorder usually coexisted with other somatic complaints, IBS per se has an obvious im- pact on living and quality of life of sufferers leading to the excessive social costs for medical seeking behavior and absenteeism. ${ }^{3-9}$ Today, IBS has been included as one of the commonly presented functional gastrointestinal disorders (FGIDs). ${ }^{1}$ It is of interest how commonly it presents in the society. In addition, knowing the IBS prevalence may estimate how it would consume the limited medical resources in the society. Western studies indicated that the population-based IBS prevalence widely ranges between $3 \%-20 \%$, whereas the most reported data range from

Received: July 16, 2010 Revised: August 18, 2010 Accepted: August 19, 2010

(c) This is an Open Access article distributed under the terms of the Creative Commons Attribution Non-Commercial License (http://creativecommons. org/licenses/by-nc/3.0) which permits unrestricted non-commercial use, distribution, and reproduction in any medium, provided the original work is properly cited.

*Correspondence: Full-Young Chang, MD

Division of Gastroenterology, Taipei Veterans General Hospital, 201 Shih-Pai Road, Section 2, Taipei, Taiwan 11217

Tel: +886-2-2875-7308, Fax: +886-2-2873-9318, E-mail: changfy@vghtpe.gov.tw

Financial support: This study was supported by a grant of Taipei Veterans General Hospital (Grant No. V99C1-084).

Conflicts of interest: None. 
$10 \%$ to $15 \% .{ }^{10,11}$ It draws a controversy why the IBS prevalence studies usually show an obvious discrimination. Actually, most prevalence study obtained data completely depend upon the response of distributed questionnaires to the studied subjects. Thereafter, the demographic characteristics in terms of evaluated population, willing of responsiveness, geographical location, criteria to define, etc always individually exist leading to the study heterogeneity and variation in the reported IBS prevalences. ${ }^{12-14}$ Of them, the employed IBS diagnostic criteria may obtain extremely distinct results for the same population. For example, Manning definition often yields a higher value compared to this of either Rome I or II definition with a gap of 2-5 folds or 2.5\%-37.0\%. ${ }^{4,14}$ Even confined to the Manning definition, the number of used disorders results in different values. ${ }^{14}$ In addition, large-scaled and questionnaire-based prevalence study does not exclude the alarm symptoms and the obtained results are unlikely to reflect the true IBS manifestations seen clinically. ${ }^{4,15,16}$ Since a characteristically biopsychosocial disorder, many social and cultural factors indeed have an apparent impact on the meaning, expression and course of FGIDs including IBS. ${ }^{17}$ Accordingly, gender, ${ }^{18-22}$ age, ${ }^{23,24}$ economic state, ${ }^{24-31}$ education level, ${ }^{24-29}$ married state, ${ }^{25,28,31}$ food, ${ }^{29-32}$ race, ${ }^{33}$ stress, ${ }^{21,22,28,31,34-36}$ climate, ${ }^{29,31}$ drugs, ${ }^{35}$ dysentery history ${ }^{29,35}$ and coexisted dyspepsia ${ }^{37}$ etc all have been addressed to be the risk factors leading to IBS.

Unfortunately, it is very hard to define that all enrolled study populations throughout the world should be harmonized with a least heterogeneity. Thus obtained IBS prevalences throughout the world would be quite variable among the studies. Besides, it is unknown whether these mentioned factors are truly existed because prevalence studies do not attempt to confirm their existence. Even some studies indicate the contradictory impact on the IBS. For instance, the same questionnaire applied to different countries may obtain the very dissimilar prevalence results, such as this conducted in Thailand and Western countries, ${ }^{38}$ another conducted in USA and 8 European countries ${ }^{39,40}$ and an early study carried out in Japan and The Netherlands, ${ }^{41}$ respectively. With regard to the IBS prevalence around the world, it has been indicated that the IBS prevalence is lower in non-western countries. ${ }^{17}$ In addition, a study based on Manning criteria indicated that the IBS prevalence of USA Asian residents was lower compared to others. ${ }^{42}$ However, this early statement meets challenge. Likewise, IBS prevalence is reported as higher as $26 \%-33 \%$ in the selected populations of the African countries. ${ }^{23,43}$ Similarly, the Japanese IBS prevalence was almost 3-fold of this of Dutch using the same questionnaire. ${ }^{41} \mathrm{Kang}^{44}$ compared East
IBS prevalences to those of West counterparts and provided the median values of $12 \%$ vs $17 \%$ in Manning criteria, $10.4 \%$ vs $9.2 \%$ in Rome I and $7.6 \%$ vs $6.0 \%$ in Rome II, respectively. He finally concluded no convincing prevalence difference existed in the East and West countries. In the same year, Gwee et $\mathrm{al}^{45}$ believed that the IBS prevalence is increasing among the Asia countries with the estimated community prevalence ranging $5.7 \%-8.6 \%$, whereas these of selected population reach up to $16 \%-45 \%$. Most recently, he reviewed the Asia IBS studies and indicated that the Asia IBS prevalence has a trend of higher in the affluent cities compared to South Asia, eg, 8.6\%-9.8\% vs $4.2 \%{ }^{46}$ Talley $^{4}$ also pointed out that IBS is prevalent in both East and West countries without necessarily to emphasize on the prevalence difference. With regard to study IBS prevalence, employed criteria should be considered since it may produce enormously different results. ${ }^{47,48}$ While the proportions of IBS subtypes may not correlate well between the used criteria. ${ }^{49,50}$ Nevertheless, it is believed that no consistent differences in sensitivity and specificity between various criteria to diagnose IBS clinically. ${ }^{51}$ Very recently, many Asia IBS prevalence studies have been published within this decade. Now we could compare the IBS prevalence data divided by various criteria in looking whether they were also comparable to this of West community.

\section{Community Population Based IBS Prevalences of Western Developed Countries}

Hungin et $\mathrm{al}^{40}$ had conducted a large-scaled study that evenly enrolled population sizes of UK, 5999; France, 5033; Germany, 5002; Italy, 5082; Holland, 5463; Belgium, 5229; Spain, 5097 and Switzerland, 5079, respectively. Their study indicated that the recommended Rome criteria obtained a value less than $5 \%$, whereas the extreme variation of prevalence values among enrolled countries still existed, eg, $11.7 \%$ in Italy and only $1.7 \%$ in German. Besides, Manning criteria defined IBS prevalence was higher compared to those of 2 Rome definitions within the same population. In order to know what is the most reported population based IBS prevalence, Table 1 illustrates 25 community-based IBS prevalences of Latin America and Western developed countries ranging from Europe, North America to Australia. Overall, 5 of 11 Manning and other criteria defined prevalence data are less than $10.5 \%$, whereas remaining 6 values are higher than this point. In addition, 14 of 21 Rome defined prevalences are within the range $2 \%-10 \%$, whereas another 7 
prevalence data are higher than $10 \%$ particularly a Canadian study based on small sample size. ${ }^{18}$ Secondly, Manning criteria based studies usually report a higher prevalence than this of Rome definition within the same population. Thirdly, the same country using the same criteria may obtain different values among various enrolled community populations. Fourthly, small-scale study often results in a higher IBS prevalence even based on community population. Finally, female predominance is invariably found no matter of used IBS definitions.

Table 1. Community Population Based Prevalences of Irritable Bowel Syndrome Among the Western Developed Countries and Latin America

\begin{tabular}{|c|c|c|c|c|c|}
\hline Author & Year of publication & Country & Sample size & Prevalence (Used criteria) & $\mathrm{M} / \mathrm{F}$ ratio \\
\hline \multirow[t]{4}{*}{ Hungin $^{40}$} & 2003 & 8 European countries & 41,984 & $6.8 \%$ (Any) & $1 / 1.69(\mathrm{NM})$ \\
\hline & & & & $6.5 \%$ (Manning) & \\
\hline & & & & $4.2 \%$ (Rome I) & \\
\hline & & & & $2.9 \%$ (Rome II) & \\
\hline Vandvik $^{19}$ & 2006 & Norway & 4,622 & $8.4 \%$ (Rome II) & $1 / 1.56$ \\
\hline Agréus $^{52}$ & 1995 & Sweden & 1,156 & $12.5 \%(\mathrm{NM})$ & $\mathrm{ND}$ \\
\hline \multirow[t]{4}{*}{ Hillilä $^{14}$} & 2004 & Finland & 3,650 & $16.2 \%$ (Manning X2) & 1/1.47 (M X2) \\
\hline & & & & 9.7\% (Manning X3) & $1 / 1.35(\mathrm{M} \mathrm{X} 3)$ \\
\hline & & & & $5.6 \%$ (Rome I) & 1/1.10 (Rome I) \\
\hline & & & & $5.1 \%$ (Rome II) & 1/1.04 (Rome II) \\
\hline \multirow[t]{2}{*}{ Hillilia $^{9}$} & 2010 & Finland & 3,650 & $15.9 \%$ (Manning) & $1 / 1.50$ \\
\hline & & & & $5.1 \%$ (Rome II) & $1 / 1.10$ \\
\hline Jones $^{53}$ & 1992 & UK & 1,620 & $22.0 \%$ (Manning) & $1 / 1.38$ \\
\hline Kennedy ${ }^{20}$ & 2000 & UK & 3,169 & $17.2 \%$ (Manning) & $1 / 2.20$ \\
\hline Wilson $^{54}$ & 2004 & UK & 4,807 & $10.5 \%$ (Rome II) & $1 / 2.12$ \\
\hline \multirow[t]{3}{*}{ Bommelaer $^{55}$} & 2004 & France & 8,221 & $2.5 \%$ (Manning) & 1/1.82 (Manning) \\
\hline & & & & $2.1 \%$ (Rome I) & 1/2.00 (Rome I) \\
\hline & & & & $1.1 \%$ (Rome II) & 1/1.44 (Rome II) \\
\hline Dapoigny $^{56}$ & 2004 & France & 20,000 & $4.7 \%$ (Rome II) & $1 / 1.54$ \\
\hline \multirow[t]{3}{*}{ Mearin $^{47}$} & 2001 & Spain & 2,000 & $10.3 \%$ (Manning) & $1 / 2.63$ \\
\hline & & & & $12.1 \%$ (Rome I) & $1 / 2.33$ \\
\hline & & & & $3.3 \%$ (Rome II) & $1 / 2.42$ \\
\hline Corazziari $^{57}$ & 2008 & Italy & 29,139 & $7.4 \%(\mathrm{NM})$ & $1 / 1.95$ \\
\hline Zagari $^{58}$ & 2010 & Italy & 1,033 & $7.1 \%$ (Rome II) & ND \\
\hline Katsinelos ${ }^{59}$ & 2009 & Greece & 2,397 & $15.7 \%$ (Rome II) & $1 / 1.31$ \\
\hline Andrews ${ }^{25}$ & 2005 & USA & 25,986 & $6.6 \%$ (Rome II) & $1 / 1.82$ \\
\hline \multirow[t]{3}{*}{ Hungin $^{39}$} & 2005 & USA & 5,009 & $10.4 \%$ (Manning) & 1/1.44 (Manning) \\
\hline & & & & $7.9 \%$ (Rome I) & 1/1.38 (Rome I) \\
\hline & & & & $6.7 \%$ (Rome II) & 1/1.36 (Rome II) \\
\hline Minocha $^{60}$ & 2006 & USA & 1,000 & $9.5 \%$ (Rome II) & NS (ND) \\
\hline Choung $^{61}$ & 2007 & USA & 2,298 & $4.4 \%$ (Rome II) & $\mathrm{ND}$ \\
\hline Jung $^{62}$ & 2010 & USA & 1,695 & $13.2 \%$ (Rome II) & $1 / 1.93$ \\
\hline \multirow[t]{2}{*}{ Thompson $^{63}$} & 2002 & Canada & 1,198 & $13.5 \%$ (Rome I) & 1/2.13 (Rome I) \\
\hline & & & & $12.1 \%$ (Rome II) & 1/1.74 (Rome II) \\
\hline $\mathrm{Li}^{18}$ & 2003 & Canada & 437 & $25.2 \%$ (Rome II) & $1 / 1.88$ \\
\hline Valerio-Ureña ${ }^{64}$ & 2010 & Mexico & 459 & $16.9 \%$ (Rome II) & $1 / 1.96$ \\
\hline Gómez Alvarez $^{65}$ & 2009 & Colombia & 558 & $19.9 \%$ (Rome III) & ND \\
\hline \multirow[t]{3}{*}{ Boyce $^{34}$} & 2000 & Australia & 2,910 & $13.6 \%$ (Manning) & 1/1.89 (Manning) \\
\hline & & & & $4.4 \%$ (Rome I) & $1 / 3.10$ (Rome I) \\
\hline & & & & $6.9 \%$ (Rome II) & 1/2.19 (Rome II) \\
\hline \multirow[t]{2}{*}{ Boyce $^{66}$} & 2006 & Australia & 762 & $4.4 \%$ (Rome I) & 1/2.98 (Rome I) \\
\hline & & & & $8.9 \%$ (Rome II) & 1/1.37 (Rome II) \\
\hline
\end{tabular}

X2, 2 diagnostic statements; X3, 3 diagnostic statements; M/F, male/female; ND, no data; NM, not mentioned; NS, not significant. 


\section{IBS Prevalences of Asia Countries}

Table 2 provides 11 IBS prevalence data of Asia countries. Of them, 6 were based on Manning definition, whereas 5 did not mention their employed definitions. Most reported prevalences are within the range $2.3 \%-11.5 \%$ but a Japanese study based on selected population obtained a higher value. ${ }^{41}$ Apart from other definitions, the median value of 6 Manning based studies is $10 \%$. Female predominance is found but not always existed. Based on Rome I criteria definition, Table 3 depicts 8 Asia IBS prevalence data plus another Korea study from Rome $\alpha$ definition. Many of them were conducted in East Asia. The range of 4 community population based prevalences is within $0.82 \%-10.4 \%$, whereas the data of 5 selected population studies are variable from overlapping to much higher. The median value of 8 Rome I definition based studies is $6.5 \%$. Female predominance remains existed. Rome II criteria based prevalence has been extensively reported in Asia, Table 4 provides 35 IBS prevalence values obtained as far as from Turkey to the most Eastern Asia countries such as Japan and Korea. Overall, 14 of 16 community population based prevalences are within $2.9 \%$ and $10.2 \%$, whereas another 2 values are higher up to about $14.0 \%{ }^{82,86}$ With regard to 19 data of selected population studies, only 7 values are less than $10.0 \%$, whereas other 12 higher values even reach up to $31.0 \%{ }^{97}$ Overall, the median value of 35 Rome II definition based studies is $8.6 \%$. Regarding the gender difference based on Rome II criteria, 22 of 31 studies reported a female predominance, whereas 5 reported male predominance and 4 reported almost equally distributed. Table 5 provides 9 Rome III criteria defined IBS prevalence data among the Asia countries. Two West Asia community-based studies indicate the values of $1.1 \%$ and $11.4 \%$, respectively. ${ }^{26,101}$

Table 2. Prevalences of Manning and Other Criteria Defined Irritable Bowel Syndrome in Asia Countries

\begin{tabular}{lllclcl}
\hline \multicolumn{1}{c}{ Author } & $\begin{array}{c}\text { Year of } \\
\text { publication }\end{array}$ & Country & Sample size & Study type & $\begin{array}{c}\text { Prevalence } \\
\text { (Used criteria) }\end{array}$ & M/F ratio \\
\hline Massarrat $^{67}$ & 1995 & Iran & 947 & Selected & $3.2 \%$ (NM) & NM \\
Shah $^{68}$ & 2001 & India & 2,549 & Community & $7.5 \%$ & NM \\
Ghoshal $^{69}$ & 2008 & India & 4,500 & Community & $4.2 \%$ (NM) & $1 / 0.93$ \\
Danivat $^{38}$ & 1988 & Thailand & 912 & Selected & $4.4 \%$ (NM) & NM \\
Ho $^{70}$ & 1998 & Singapore & 696 & Community & $2.3 \%$ (Local) & M $=$ F (ND) \\
Chen $^{71}$ & 2000 & Singapore & 271 & Community & $3.2 \%(\mathrm{NM})$ & $1 / 1.13$ \\
Gwee $^{24}$ & 2004 & Singapore & 2,276 & Community & $11.0 \%$ & $1 / 1.33$ \\
Lau $^{72}$ & 2002 & Hong Kong & 1,298 & Community & $10.0 \%$ & NM \\
Pan & 2000 & China & 2,486 & Community & $7.3 \%$ & $1 / 1.15$ \\
Xiong $^{35}$ & 2004 & China & 4,178 & Community & $11.5 \%$ & $1 / 1.34$ \\
Schlemper & 1993 & Japan & 231 & Selected & $25.0 \%$ & $1 / 1.35$
\end{tabular}

$\mathrm{M} / \mathrm{F}$, male/female; ND, no data; NM, not mentioned.

Table 3. Prevalences of Rome I and $\alpha$ Criteria Defined Irritable Bowel Syndrome in Asia Countries

\begin{tabular}{|c|c|c|c|c|c|c|}
\hline Author & $\begin{array}{c}\text { Year of } \\
\text { publication }\end{array}$ & Country & Sample size & Study type & Prevalence & $\mathrm{M} / \mathrm{F}$ ratio \\
\hline Zuckerman $^{73}$ & 2006 & Vietnam & 411 & Selected & $7.20 \%$ & $1 / 1.92$ \\
\hline $\operatorname{Tan}^{36}$ & 2003 & Malaysia & 533 & Selected & $15.80 \%$ & $1 / 1.59$ \\
\hline Gwee $^{24}$ & 2004 & Singapore & 2,276 & Community & $10.40 \%$ & $1 / 1.30$ \\
\hline $\mathrm{Lau}^{72}$ & 2002 & Hong Kong & 1,298 & Community & $6.00 \%$ & $\mathrm{NM}$ \\
\hline Cheung $^{74}$ & 2007 & Hong Kong & 1,649 & Community & $4.00 \%$ & $\mathrm{M}=\mathrm{F}(\mathrm{ND})$ \\
\hline $\operatorname{Pan}^{29}$ & 2000 & China & 2,486 & Community & $0.82 \%$ & NM \\
\hline $\mathrm{Lu}^{75}$ & 2003 & Taiwan & 2,018 & Selected & $17.50 \%$ & $\mathrm{NM}$ \\
\hline $\mathrm{Kim}^{30}$ & 2005 & Korea & 1,717 & Selected & $5.70 \%($ Rome $\alpha)$ & $1 / 1.27$ \\
\hline Kawamura $^{76}$ & 2001 & Japan & 2,263 & Selected & $6.50 \%$ & NM \\
\hline
\end{tabular}

$\mathrm{M} / \mathrm{F}$, male/female; ND, no data; NM, not mentioned. 
Table 4. Prevalences of Rome II Criteria Defined Irritable Bowel Syndrome in Asia Countries

\begin{tabular}{|c|c|c|c|c|c|c|}
\hline Author & Year of publication & Country & Sample size & Study type & Prevalence & $\mathrm{M} / \mathrm{F}$ ratio \\
\hline Sperber $^{26}$ & 2007 & Israel & 1,000 & Community & $2.9 \%$ & $1 / 2.06$ \\
\hline Celebi $^{27}$ & 2004 & Turkey & 1,766 & Selected & $6.3 \%$ & $1 / 1.48$ \\
\hline Yilmaz $^{77}$ & 2005 & Turkey & 3,000 & Community & $10.2 \%$ & $1 / 1.55$ \\
\hline Basaranoglu $^{78}$ & 2008 & Turkey & 707 & Selected & $8.6 \%$ & $\mathrm{M}=\mathrm{F}(\mathrm{ND})$ \\
\hline Hoseini-Asi ${ }^{.79}$ & 2003 & Iran & 4,726 & Community & $5.8 \%$ & $1 / 1.17$ \\
\hline Sohrabi ${ }^{80}$ & 2010 & Iran & 1,436 & Selected & $4.1 \%$ & $1 / 2.19$ \\
\hline Yarandi ${ }^{81}$ & 2010 & Iran & 6,476 & Selected & $21.9 \%$ & $1 / 1.58$ \\
\hline $\mathrm{Jafri}^{82}$ & 2007 & Pakistan & 1,167 & Community & $14.0 \%$ & $\mathrm{M}>\mathrm{F}(\mathrm{ND})$ \\
\hline Husain $^{28}$ & 2008 & Pakistan & 880 & Selected & $13.3 \%$ & $1 / 1.02$ \\
\hline Masud $^{83}$ & 2001 & Bangladesh & 2,426 & Community & $8.5 \%$ & $1 / 1.84$ \\
\hline Perveen $^{84}$ & 2009 & Bangladesh & 1,503 & Community & $7.7 \%$ & $1 / 1.28$ \\
\hline Devanarayana $^{85}$ & 2010 & Sri Lanka & 427 & Selected & $2.8 \%$ & NM \\
\hline Rajendra $^{86}$ & 2004 & Malaysia & 949 & Community & $15.6 \%$ & $1 / 1.40$ \\
\hline Gwee $^{24}$ & 2004 & Singapore & 2,276 & Community & $8.6 \%$ & $1 / 1.21$ \\
\hline $\mathrm{Lau}^{72}$ & 2002 & Hong Kong & 1,298 & Community & $3.6 \%$ & $1 / 1.06$ \\
\hline $\mathrm{Kwan}^{87}$ & 2002 & Hong Kong & 1,000 & Community & $6.6 \%$ & $1 / 1.30$ \\
\hline $\mathrm{Lee}^{88}$ & 2009 & Hong Kong & 2,005 & Community & $5.4 \%$ & $1 / 1.35$ \\
\hline Xiong $^{35}$ & 2004 & China & 4,178 & Community & $5.7 \%$ & $1 / 1.25$ \\
\hline Dong $^{31}$ & 2005 & China & 5,403 & Selected & $13.8 \%$ & $1 / 1.80$ \\
\hline Wang $^{89}$ & 2007 & China & 3,014 & Selected & $18.5 \%$ & $\mathrm{M}=\mathrm{F}(\mathrm{ND})$ \\
\hline $\mathrm{Dai}^{90}$ & 2008 & China & 1,147 & Selected & $4.7 \%$ & $1 / 1.34$ \\
\hline Shen $^{91}$ & 2009 & China & 491 & Selected & $15.7 \%$ & $1 / 1.16$ \\
\hline Zhao $^{92}$ & 2010 & China & 16,091 & Community & $4.6 \%$ & $1 / 1.22$ \\
\hline $\mathrm{Lu}^{75}$ & 2003 & Taiwan & 2,018 & Selected & $22.1 \%$ & $1 / 0.64, \mathrm{NS}$ \\
\hline $\operatorname{Han}^{32}$ & 2006 & Korea & 1,066 & Community & $6.6 \%$ & $1 / 0.85$ \\
\hline Park $^{21}$ & 2008 & Korea & 1,124 & Selected & $16.8 \%$ & $1 / 1.36$ \\
\hline $\mathrm{Lee}^{93}$ & 2009 & Korea & 1,443 & Selected & $9.6 \%$ & NM \\
\hline Son $^{94}$ & 2009 & Korea & 405 & Selected & $25.7 \%$ & Only enrolled girls \\
\hline Park $^{95}$ & 2010 & Korea & 1,009 & Community & $8.0 \%$ & $1 / 1.50$ \\
\hline Kumano $^{22}$ & 2004 & Japan & 4,000 & Community & $6.1 \%$ & $1 / 1.73$ \\
\hline Shiotani ${ }^{96}$ & 2006 & Japan & 2,495 & Selected & $10.7 \%$ & $1 / 2.04$ \\
\hline Shinozaki $^{97}$ & 2008 & Japan & 633 & Selected & $31.0 \%$ & $1 / 1.24$ \\
\hline Hori $^{98}$ & 2009 & Japan & 181 & Selected & $22.1 \%$ & NM \\
\hline Okumura $^{99}$ & 2010 & Japan & 5,813 & Selected & $1.2 \%$ & $1 / 0.92$ \\
\hline $\mathrm{Kaji}^{100}$ & 2010 & Japan & 2,680 & Selected & $14.2 \%$ & NM \\
\hline
\end{tabular}

$\mathrm{M} / \mathrm{F}$, male/female; ND, no data; NM, not mentioned; NS, not significant.

A Korea community study provides prevalence of $9.0 \%{ }^{98}$ whereas 6 selected population based studies are very variable ranging $7.0 \%-21.9 \%$. The median value of 9 Rome III definition based studies is $10.1 \%$. Female predominance is also found among 7 reports.

Summarized together, the reported IBS prevalences of Tables 2-5 are very variable with a range $0.82 \%-31.0 \%$ among the Asia countries. However, these data are likely to indicate that the most Asia community prevalences are within the range 1\%-10\% and lower than these of selected populations as well as previous comments. ${ }^{45,105,106}$ Similar to Table 1 , the same country using the same criteria usually yields dissociated results among the different study populations. On the other hand, the same population always results in different prevalences defined by various criteria. Within the same population, the prevalence orders are usually first higher based on Manning criteria, then followed by Rome I criteria and finally reported in Rome II criteria. This ranking characteristic is also to agree with Asia consensus. ${ }^{106}$ Occasionally, few studies in Asia and developed countries contradictorily indicated the higher value based on Rome II than Rome I. ${ }^{34,75}$ With regard to Rome III criteria, it is apparently higher than Rome II reported in Israeli, Chinese and Korean studies, respectively, ${ }^{26,90,95}$ 
Table 5. Prevalences of Rome III Defined Irritable Bowel Syndrome in Asia Countries

\begin{tabular}{lclclrc}
\hline \multicolumn{1}{c}{ Author } & $\begin{array}{c}\text { Year of } \\
\text { publication }\end{array}$ & Country & Sample size & Study type & Prevalence & M/F ratio \\
\hline Sperber $^{26}$ & 2007 & Israel & 1,000 & Community & $11.4 \%$ & $1 / 1.37$ \\
Khoshkrood-Mansoori $^{101}$ & 2009 & Iran & 18,180 & Community & $1.1 \%$ & $1 / 2.31$ \\
Yarandi $^{81}$ & 2010 & Iran & 6,476 & Selected & $21.9 \%$ & $1 / 1.58$ \\
Devanarayana $^{85}$ & 2010 & Sri Lanka & 427 & Selected & $7.0 \%$ & $1 / 1.15$ \\
Dai $^{90}$ & 2008 & China & 1,147 & Selected & $10.4 \%$ & $1 / 1.85$ \\
Wang $^{102}$ & 2008 & China & 3,014 & Selected & $15.9 \%$ & $1 / 1.17$ \\
Park $^{95}$ & 2010 & Korea & 1,009 & Community & $9.0 \%$ & $1 / 1.57$ \\
Nam $^{103}$ & 2010 & Korea & 4,296 & Selected & $9.1 \%$ & $1 / 1.04$ \\
Noh $^{104}$ & 2010 & Korea & 2,388 & Selected & $10.1 \%$ & NM \\
\hline
\end{tabular}

$\mathrm{M} / \mathrm{F}$, male/female; NM, not mentioned.

whereas another study carried out in a selected Chinese population showed comparable in both definitions. ${ }^{89,102}$ In contrast, an Iran study yields a much lower prevalence by way of Rome III criteria. ${ }^{101}$ Most importantly, the IBS prevalence studies conducted on multi-ethnic countries such as Malaysia and Singapore clearly pointed out no difference existed among various ethnic populations. ${ }^{24,36,70,86}$ Similarly, the IBS prevalence of Chinese minority was not different from this of Han ethnic. ${ }^{31}$ Based on these observations and comparisons, we as well as the Asia consensus ${ }^{106}$ are strongly to conclude that the current Asia IBS prevalence is at least equal to the Western countries.

\section{Gender Difference of Asia Countries}

Gender factor may play an impact on the FGIDs including IBS. As previously indicated that female gender has been one of factors leading to IBS and females usually report more FGID symptoms. ${ }^{63}$ Perhaps it means that the visceral perception is determined by the sex. ${ }^{17}$ Female IBS subjects also have lower authority over decisions at work. ${ }^{107}$ Besides, Caucasian females easily suffer from IBS symptoms compared to males or African American females. ${ }^{33}$ Based on the current IBS diagnostic criteria, higher positive predictive value is often obtained for females, whereas a negative predictive value exists for males. ${ }^{108}$ Accordingly, it is unknown whether these gender-related specificities account for the female predominance in many IBS studies. Clinically, constipation predominant IBS (C-IBS) is commonly seen among the females, whereas males usually manifest diarrhea-predominant IBS (D-IBS)..$^{19,25,90,109,110}$ In addition, IBS is common among the Vietnamese females but no significant gender effect on some bowel symptoms in terms of stool frequency, consistency and bloating is observed. ${ }^{73}$ Table 1 depicts the IBS male/female ratio among the developed countries. Almost all except a USA study indicate the female predominance, irrespectively of used criteria. ${ }^{60}$ Interestingly, a Finnish study showed female predominance in Manning definition and gradually became equal in Rome II definition. ${ }^{14}$ For the African IBS, female predominance is not reported yet. ${ }^{23,43}$ Regarding the Asian IBS gender issue, Gwee et $\mathrm{al}^{45}$ early pointed out lack of female predominance except the existed female predominance in Japan. Recent Asia IBS consensus also concludes no obvious female predominance existed in many Asia studies. ${ }^{106}$ Tables 2-5 provide the sex ratios among cited Asia studies. The ratios are widely variable ranging from equal to 2 -fold of female predominance. Even the same country may not obtain the similar gender ratio among various reports, eg, Pakistan, Singapore, China and Korea, respectively. Accordingly, the female predominance looks to be commonly existed in Near East, Bangladesh, Southeast Asia, China, Japan and finally some but not all Korea studies, whereas the remaining countries just show similar or diverse ratio. With regard to the used criteria, a Korea study indicates that female subjects showed a higher prevalence than male subjects under Rome III but not under Rome II criteria. ${ }^{95}$ Summarized together, the IBS female predominance is not uniquely existed among all Asia countries.

\section{Subtypes of IBS in Asia}

The IBS subtypes are mainly divided according to the bowel movement (BM) frequency and stool consistency of the presenting subjects. Consequently, it looks important to know what is the so-called normal bowel habit in the general population. Among the Asia countries, many studies already provide the BM parameters. For instance, subjects with non-complaint of lower abdo- 
men symptoms in India usually have the BM as 1-2 times/day. ${ }^{69}$ Over $90 \%$ people of Thailand and Singapore have the BM as 3 times/day to 3 times/wk, while nearly $60 \%$ of them pass once daily. ${ }^{38,70}$ Koreans also exhibit this BM pattern and $41 \%$ of them report once daily. ${ }^{30}$ Meanwhile, $84 \%$ Chinese non-patient community subjects report BM once daily with a mean value 7 times/wk. Besides, $90 \%$ of them pass formed soft stool, $77 \%$ finish it in the morning and only $3.8 \%$ show constipation and $1.1 \%$ as diarrhea, respectively. ${ }^{111}$ It can be concluded that the BM ranging 3 times/day to 3 times/wk is acceptable as normal for most Asia people.

Regarding the IBS subtypes, this subjective category remains difficult in clinical practice. Because the restrictive character of Rome II definition and alternating-IBS (A-IBS) being really unknown as part of C- or D-IBS, most IBS are perhaps undiagnosed. ${ }^{12,39}$ Surprisingly, even some A-IBS subjects think that their BM is normal despite the alternating expression. ${ }^{24}$ Thereafter, who such as physician or patient himself or what criteria should determine the subtype correctly? In addition, IBS subtypes are likely depending upon heterogeneity in terms of evaluated population, gender, geographical location and criteria to define. $^{12,19,22,40,44,90,108,110}$ For example, the subtype correlation between Rome II and III has been poor because the apparently existed drawback in the categories of A-IBS, mixed-IBS and un-

Table 6. Percentile Distribution of Reported Subtypes in Subjects With Irritable Bowel Syndrome

\begin{tabular}{|c|c|c|c|c|c|c|c|}
\hline Study & Country & Sample & $\begin{array}{l}\text { Criteria } \\
\text { size }\end{array}$ & $\begin{array}{c}\text { D-IBS } \\
(\%)\end{array}$ & $\begin{array}{c}\text { C-IBS } \\
(\%)\end{array}$ & $\begin{array}{c}\text { A (M)-IBS } \\
(\%)\end{array}$ & $\begin{array}{c}\text { U-IBS } \\
(\%)\end{array}$ \\
\hline \multicolumn{8}{|l|}{ Western and Latin } \\
\hline \multicolumn{8}{|l|}{ America countries } \\
\hline Hungin $^{40} 2003$ & $\begin{array}{l}8 \text { European } \\
\text { countries }\end{array}$ & $41,984^{\mathrm{a}}$ & Rome II & 21.0 & 16.0 & 63.0 & - \\
\hline Vandvik $^{19} 2006$ & Norway & $4,622^{\mathrm{a}}$ & Rome II & 23.0 & 24.0 & 53.0 & - \\
\hline \multirow[t]{2}{*}{ Ersryd $^{49} 2007$} & Sweden & 249 & Rome II & 36.5 & 18.1 & 45.4 & - \\
\hline & & & Rome III & 39.0 & 31.0 & 63.0 & 23.7 \\
\hline \multirow{4}{*}{ Hillilä $^{14} 2004$} & Finland & $3,650^{\mathrm{a}}$ & Manning X2 & 41.2 & 22.7 & NM & NM \\
\hline & & & Manning X3 & 47.6 & 18.7 & $\mathrm{NM}$ & $\mathrm{NM}$ \\
\hline & & & Rome I & 53.9 & 21.5 & $\mathrm{NM}$ & - \\
\hline & & & Rome II & 56.5 & 16.3 & $\mathrm{NM}$ & - \\
\hline Jones $^{53} 1992$ & UK & $1,620^{\mathrm{a}}$ & Manning & 58.0 & 48.0 & 27.0 (Both) & 21.0 (Neither) \\
\hline Wilson $^{54} 2004$ & UK & $4,807^{\mathrm{a}}$ & Rome II & 25.4 & 24.1 & 46.7 & - \\
\hline Dapoigny ${ }^{56} 2004$ & France & $20,000^{\mathrm{a}}$ & Rome II & 36.0 & 29.0 & 31.0 & - \\
\hline Katsinelos $^{59} 2009$ & Greece & $2,397^{\mathrm{a}}$ & Rome II & 36.5 & 44.2 & 19.3 & - \\
\hline Andrews ${ }^{25} 2005$ & USA & $25,986^{\mathrm{a}}$ & Rome II & 53.0 & 19.7 & 25.8 & - \\
\hline \multirow{2}{*}{ Hungin $^{39} 2005$} & USA & $5,009^{\mathrm{a}}$ & Rome I & 20.7 & 15.5 & 63.8 & - \\
\hline & & & Rome II & 22.6 & 16.1 & 61.3 & - \\
\hline Valerio-Ureña ${ }^{64} 2010$ & Mexico & $459^{\mathrm{a}}$ & Rome II & 28.2 & 50.0 & 21.8 & - \\
\hline Schmulson ${ }^{113} 2010$ & Mexico & 1,021 & Rome II & 28.5 & 27.8 & 43.7 & - \\
\hline \multicolumn{8}{|l|}{ Asia countries } \\
\hline \multirow[t]{2}{*}{ Sperber $^{26} 2007$} & Israel & $1,000^{\mathrm{a}}$ & Rome II & 32.1 & 57.1 & 10.7 & - \\
\hline & & & Rome III & 16.1 & 36.6 & 47.3 & NM \\
\hline Celebi $^{27} 2004$ & Turkey & 1,766 & Rome II & 36.0 & 52.3 & 11.7 & - \\
\hline Yilmaz $^{77} 2005$ & Turkey & $3,000^{\mathrm{a}}$ & Rome II & 48.1 & 38.9 & - & 13.0 \\
\hline $\begin{array}{l}\text { Khoshkrood- } \\
\text { Mansoori }^{101} 2009\end{array}$ & Iran & $18,180^{\mathrm{a}}$ & Rome III & 18.0 & 52.0 & 8.0 & $\mathrm{NM}$ \\
\hline Husain $^{28} 2008$ & Pakistan & 880 & Rome II & 29.3 & 50.4 & 20.3 & - \\
\hline Ghoshal $^{69} 2008$ & India & $4,500^{\mathrm{a}}$ & $\mathrm{NM}$ & 4.0 & 39.0 & - & 57.0 \\
\hline Masud $^{83} 2001$ & Bangladesh & $2,426^{\mathrm{a}}$ & Rome II & 0.8 & 16.0 & 19.2 & 63.9 \\
\hline Perveen $^{84} 2009$ & Bangladesh & $1,503^{\mathrm{a}}$ & Rome II & 50.0 & 16.4 & 7.8 & 25.9 \\
\hline
\end{tabular}

${ }^{a}$ Community based study.

X2, 2 diagnostic statements; X3, 3 diagnostic statements; IBS, irritable bowel syndrome; D-IBS, diarrhea predominant-IBS; C-IBS, constipation predominant-IBS; A-IBS, alternating-IBS; M-IBS, mixed-IBS; U-IBS, un-subtyped-IBS; NM, not mentioned. 
Table 6. Continued

\begin{tabular}{|c|c|c|c|c|c|c|c|}
\hline Study & Country & Sample & $\begin{array}{l}\text { Criteria } \\
\text { size }\end{array}$ & $\begin{array}{c}\text { D-IBS } \\
(\%)\end{array}$ & $\begin{array}{c}\text { C-IBS } \\
(\%)\end{array}$ & $\begin{array}{c}\text { A (M)-IBS } \\
(\%)\end{array}$ & $\begin{array}{c}\text { U-IBS } \\
(\%)\end{array}$ \\
\hline \multirow[t]{2}{*}{ Devanarayana $^{85} 2010$} & Sri Lanka & 427 & Rome II & 25.0 & 33.3 & 16.7 & 25.0 \\
\hline & & & Rome III & 26.7 & 26.7 & 33.3 & 13.3 \\
\hline $\operatorname{Tan}^{36} 2003$ & Malaysia & 533 & Rome I & 7.1 & 77.4 & - & 15.5 \\
\hline Rajendra $^{86} 2004$ & Malaysia & $949^{\mathrm{a}}$ & Rome II & 35.1 & 36.5 & 28.4 & - \\
\hline Gwee $^{24} 2004$ & Singapore & $2,276^{\mathrm{a}}$ & Rome II & 12.8 & 51.0 & $\mathrm{NM}$ & - \\
\hline $\mathrm{Kwan}^{87} 2002$ & Hong Kong & $1,000^{\mathrm{a}}$ & Rome II & 27.0 & 17.0 & - & 56.0 \\
\hline $\mathrm{Si}^{110} 2004$ & China & 662 & Rome II & 47.7 & 20.0 & 32.3 & - \\
\hline Xiong $^{35} 2004$ & China & $4178^{\mathrm{a}}$ & Rome II & 74.1 & 15.1 & 10.8 & - \\
\hline \multirow[t]{2}{*}{ Wang $^{89} 2007$} & China & 3,014 & Rome II & 38.2 & 33.2 & 28.7 & - \\
\hline & & & Rome III & 32.7 & 27.9 & 6.7 & 32.7 \\
\hline Dai $^{90} 2008$ & China & 1,147 & Rome III & 24.3 & 14.7 & 48.0 & 13.0 \\
\hline Wang $^{102} 2009$ & China & 3,014 & Rome III & 32.7 & 27.9 & 6.7 & 32.7 \\
\hline Zhao $^{92} 2010$ & China & $16,091^{a}$ & Rome II & 37.7 & 24.9 & 37.4 & - \\
\hline $\mathrm{Kim}^{30} 2005$ & Korea & 1,717 & Rome $\alpha$ & 22.5 & 24.5 & - & 52.0 \\
\hline $\operatorname{Han}^{32} 2006$ & Korea & $1,066^{\mathrm{a}}$ & Rome II & 32.8 & 24.3 & 42.9 & - \\
\hline Son $^{94} 2009$ & Korea & 405 & Rome II & 26.9 & 34.5 & 38.8 & - \\
\hline \multirow[t]{2}{*}{$\operatorname{Park}^{95} 2010$} & Korea & $1,009^{\mathrm{a}}$ & Rome II & 27.2 & 37.0 & 25.9 & 9.9 \\
\hline & & & Rome III & 29.7 & 36.3 & 22.0 & 12.1 \\
\hline $\mathrm{Noh}^{104} 2010$ & Korea & 2,388 & Rome III & 54.4 & 12.0 & 12.4 & $\mathrm{NM}$ \\
\hline Kumano $^{22} 2004$ & Japan & $4,000^{\mathrm{a}}$ & Rome II & 31.1 & 41.0 & 27.9 & - \\
\hline Shiotani $^{96} 2006$ & Japan & 2,495 & Rome II & 43.7 & 47.8 & - & 8.6 \\
\hline Shinozaki ${ }^{97} 2008$ & Japan & 633 & Rome II & 29.6 & 21.4 & 49.0 & - \\
\hline Hori $^{98} 2009$ & Japan & 181 & Rome II & 65.0 & 35.0 & - & - \\
\hline
\end{tabular}

${ }^{\text {a }}$ Community based study.

X2, 2 diagnostic statements; X3, 3 diagnostic statements; IBS, irritable bowel syndrome; D-IBS, diarrhea predominant-IBS; C-IBS, constipation predominant-IBS; A-IBS, alternating-IBS; M-IBS, mixed-IBS; U-IBS, un-subtyped-IBS; NM, not mentioned.

subtyped-IBS, respectively. ${ }^{49,90}$ Finally, a review indicates that USA population-based studies found similar distribution of C-IBS, D-IBS and A-IBS, whereas European studies showed either C-IBS or A-IBS as the most prevalent subtypes. More interestingly, primary care office-based studies showed A-IBS as the most prevalent group, while the specialist office-based studies found either C-IBS or D-IBS as the most frequently reported subtype. ${ }^{12}$ It is likely the different attitude to acknowledge IBS between primary care and gastroenterologists. For example, not more than $50 \%$ of primary care physicians could reach the consensus, while cultural differences in the description of key symptoms is responsible for this discrimination. ${ }^{112}$ Table 6 depicts the percentile distribution of IBS subtypes in 12 Western and 28 Asian countries. Among the developed countries, there is quite variable in the distribution of 3 main subtypes. Apart from Manning definition, it looks that the subtype proportions of C-IBS and D-IBS usually range 15\%-55\%, whereas this of A-IBS ranges higher as $20 \%-65 \%$. The subtype correlation between Rome I and II is optimal but is not well existed between
Rome II and III. For the Asian countries, the proportions of D-IBS distribute widely from $0.8 \%$ to $74.1 \%$, C-IBS proportion ranges $12.0 \%-77.4 \%$, whereas A-IBS appears less common with range 6.7\%-49.0\%. Of 8 Rome III criteria based studies showing subtypes, 5 report unsubtyped-IBS with proportion ranged 12.1\%-32.7\%. Apart a Korea study, ${ }^{95}$ the subtype correlation between Rome II and III is poor as well as this of Western studies. Summarized together, IBS subtype proportions are very variable among the Asia studies. It is likely to mean that the clear and precise differentiation of various subtypes to fit BM stool form and occupied duration remains very subjective both for the patients and physicians themselves.

In conclusions, current Asia IBS prevalence is at least equal to the Western countries. Female predominant prevalence in Asia is common but not uniquely existed, while the proportions of IBS subtypes in Asia are too variable to find a rule. 


\section{References}

1. Drossman DA. The functional gastrointestinal disorders and the Rome III process. Gastroenterology 2006;130:1377-1390.

2. Kellow JE, Azpiroz F, Delvaux M, et al. Applied principles of neurogastroenterology: physiology/motility sensation. Gastroenterology 2006;130:1412-1420.

3. Whitehead WE, Palsson OS, Levy RR, Feld AD, Turner M, Von Korff M. Comorbidity in irritable bowel syndrome. Am J Gastroenterol 2007;102:2767-2776.

4. Talley NJ. Functional gastrointestinal disorders as a public health problem. Neurogastroenterol Motil 2008;20(suppl 1):121-129.

5. Graham DP, Savas L, White D, et al. Irritable bowel syndrome symptoms and health related quality of life in female veterans. Aliment Pharmacol Ther 2010;31:261-273.

6. Nyrop KA, Palsson OS, Levy RL, et al. Costs of health care for irritable bowel syndrome, chronic constipation, functional diarrhoea and functional abdominal pain. Aliment Pharmacol Ther 2007;26: 237-248.

7. Chang JY, Locke GR 3rd, McNally MA, et al. Impact of functional gastrointestinal disorders on survival in the community. Am J Gastroenterol 2010;105:822-832.

8. Lu CL, Chang FY, Chen CY, Luo JC, Lee SD. Significance of Rome II-defined functional constipation in Taiwan and comparison with constipation-predominant irritable bowel syndrome. Aliment Pharmacol Ther 2006;24:429-438.

9. Hillï̈ MT, Färkkïä NJ, Färkkilä MA. Societal costs for irritable bowel syndrome - a population based study. Scand J Gastroenterol 2010;45:582-591.

10. Oberndorff-Klein Woolthuis AH, Brummer RJ, de Wit NJ, Muris JW, Stockbrügger RW. Irritable bowel syndrome in general practice: an overview. Scand J Gastroenterol 2004;241(suppl):17-22.

11. Saito YA, Schoenfeld P, Locke GR 3rd. The epidemiology of irritable bowel syndrome in North America: a systematic review. Am J Gastroenterol 2002;97:1910-1915.

12. Guilera M, Balboa A, Mearin F. Bowel habit subtypes and temporal patterns in irritable bowel syndrome: systematic review. Am J Gastroenterol 2005;100:1174-1184.

13. Spiller R, Aziz Q, Creed F, et al. Guidelines on the irritable bowel syndrome: mechanisms and practical management. Gut 2007;56: 1770-1798.

14. Hillilä MT, Färkkilä MA. Prevalence of irritable bowel syndrome according to different diagnostic criteria in a non-selected adult population. Aliment Pharmacol Ther 2004;20:339-345.

15. Hammer J, Eslick GD, Howell SC, Altiparmak E, Talley NJ. Diagnostic yield of alarm features in irritable bowel syndrome and functional dyspepsia. Gut 2004;53:666-672.

16. Olden KW. Diagnosis of irritable bowel syndrome. Gastroenterology 2002;122:1701-1714.

17. Chang L, Toner BB, Fukudo S, et al. Gender, age, society, culture, and the patient's perspective in the functional gastrointestinal disorders. Gastroenterology 2006;130:1435-1446.

18. Li FX, Patten SB, Hilsden RJ, Sutherland LR. Irritable bowel syndrome and health-related quality of life: a population-based study in
Calgary, Alberta. Can J Gastroenterol 2003;17:259-263.

19. Vandvik PO, Lvdersen S, Farup PG. Prevalence, comorbidity and impact of irritable bowel syndrome in Norway. Scand J Gastroenterol 2006;41:650-656.

20. Kennedy TM, Jones RH. Epidemiology of cholecystectomy and irritable bowel syndrome in a UK population. Br J Surg 2000;87: 1658-1663.

21. Park KS, Ahn SH, Hwang JS, et al. A survey about irritable bowel syndrome in South Korea: prevalence and observable organic abnormalities in IBS patients. Dig Dis Sci 2008;53:704-711.

22. Kumano H, Kaiya H, Yoshiuchi K, Yamanaka G, Sasaki T, Kuboki T. Comorbility of irritable bowel syndrome, panic disorder, and agoraphobia in a Japanese representative sample. Am J Gastroenterol 2004;99:370-376.

23. Ladep NG, Okeke EN, Samaila AA, et al. Irritable bowel syndrome among patients attending General Outpatients' clinics in Jos, Nigeria. Eur J Gastroenterol Hepatol 2007;19:795-799.

24. Gwee KA, Wee S, Wong ML, Png DJ. The prevalence, symptom characteristics, and impact of irritable bowel syndrome in an Asian urban community. Am J Gastroenterol 2004;99:924-931.

25. Andrews EB, Eaton SC, Hollis KA, et al. Prevalence and demographics of irritable bowel syndrome: results from a large web-based survey. Aliment Pharmacol Ther 2005;22:935-942.

26. Sperber AD, Shyartzman P, Friqer M, Fich A. A comparative reappraisal of Rome II and Rome III diagnostic criteria: are we getting closer to the "true" prevalence of irritable bowel syndrome? Eur J Gastroenterol Hepatol 2007;19:441-447.

27. Celebi S, Acik Y, Deveci SE, et al. Epidemiological features of irritable bowel syndrome in a Turkish urban society. J Gastroenterol Hepatol 2004;19:738-743.

28. Husain N, Chaudhry IB, Jafri F, Niaz SK, Tomenson B, Creed F. A population-based study of irritable bowel syndrome in a non-Western population. Neurogastroenterol Motil 2008;20:10221029.

29. Pan G, Lu S, Ke M, Han S, Guo H, Fang X. Epidemiologic study of the irritable bowel syndrome in Beijing: stratified randomized study by cluster sampling. Chin Med J (Engl) 2000;113:35-39.

30. Kim YJ, Ban DJ. Prevalence of irritable bowel syndrome, influence of lifestyle factors and bowel habits in Korean college students. Int J Nurs Stud 2005;42:247-254.

31. Dong L, Dingguo L, Xiaoxing X, Hanming L. An epidemiologic study of irritable bowel syndrome in adolescents and children in China: a school-based study. Pediatrics 2005;116:e393-e396.

32. Han SH, Lee OY, Bae SC, et al. Prevalence of irritable bowel syndrome in Korea: population-based survey using the Rome II criteria. J Gastroenterol Hepatol 2006;21:1687-1692.

33. Wigington WC, Johnson WD, Minocha A. Epidemiology of irritable bowel syndrome among African Americans as compared with whites: a population-based study. Clin Gastroenterol Hepatol 2005; 3:647-653

34. Boyce PM, Koloski NA, Talley NJ. Irritable bowel syndrome according to varying diagnostic criteria: are the new Rome II criteria unnecessarily restrictive for research and practice? Am J Gastroenterol 2000;95:3176-3183.

35. Xiong LS, Chen MH, Chen HX, Xu AG, Wang WA, Hu PJ. A population-based epidemiologic study of irritable bowel syndrome 
in South China: stratified randomized study by cluster sampling. Aliment Pharmacol Ther 2004;19:1217-1224.

36. Tan YM, Goh KL, Muhidayah R, Ooi CL, Salem O. Prevalence of irritable bowel syndrome in young adult Malaysians: a survey among medical students. J Gastroenterol Hepatol 2003;18:14121416.

37. Ford AC, Marwaha A, Lim A, Moayyedi P. Systematic review and meta-analysis of the prevalence of irritable bowel syndrome in individuals with dyspepsia. Clin Gastroenterol Hepatol 2010;8:401409.

38. Danivat D, Tankeyoon M, Sriratanaban A. Prevalence of irritable bowel syndrome in non-Western population. Br Med J (Clin Res Ed) 1988;296:1710.

39. Hungin AP, Chang L, Locke GR, Dennis EH, Barghout V. Irritable bowel syndrome in the United States: prevalence, symptom patterns and impact. Aliment Pharmacol Ther 2005;21:1365-1375.

40. Hungin AP, Whorwell PJ, Tack J, Mearin F. The prevalence, patterns and impact of irritable bowel syndrome: an international survey of 40,000 subjects. Aliment Pharmacol Ther 2003;17:643-650.

41. Schlemper RJ, van der Werf SDJ, Vandenbroucke JP, Biemond I, Lambers CB. Peptic ulcer, non-ulcer dyspepsia and irritable bowel syndrome in The Netherlands and Japan. Scand J Gastroenterol 1993;200(suppl):33-41.

42. Longstreth GF, Wolde-Tsadik G. Irritable bowel-type symptoms in HMO examinees. Prevalence, demographic, and clinical correlates. Dig Dis Sci 1993;38:1581-1589.

43. Okeke EN, Agaba EI, Gwamzhi L, Achinge GI, Angbazo D, Malu AO. Prevalence of irritable bowel syndrome in a Nigerian student population. Afr J Med Med Sci 2005;34:33-36.

44. Kang JY. Systemic review: the influence of geography and ethnicity in irritable bowel syndrome. Aliment Pharmacol Ther 2005;21: 663-676.

45. Gwee KA. Irritable bowel syndrome in developing countries - a disorder of civilization or colonization? Neurogastroenterol Motil 2005; 17:317-324

46. Gwee KA, Lu CL, Ghoshal UC. Epidemiology of irritable bowel syndrome in Asia: something old, something new, something borrowed. J Gastroenterol Hepatol 2009;24:1601-1607.

47. Mearin F, Badía X, Balboa A, et al. Irritable bowel syndrome prevalence varies enormously depending on the employed diagnostic criteria: comparison of Rome II versus previous criteria in a general population. Scand J Gastroenterol 2001;36:1155-1161.

48. Mearin F, Roset M, Badía X, et al. Splitting irritable bowel syndrome: from original Rome to Rome II criteria. Am J Gastroenterol 2004;99:122-130.

49. Ersryd A, Posserud I, Abrahamsson H, Simren M. Subtyping the irritable bowel syndrome by predominant bowel habit: Rome II versus Rome III. Aliment Pharmacol Ther 2007;26:953-961.

50. Dorn SD, Morris CB, Hu Y, et al. Irritable bowel syndrome subtypes defined by Rome II and Rome III criteria are similar. J Clin Gastroenterol 2009;43:214-220.

51. Whitehead WE, Drossman DA. Validation of symptom-based diagnostic criteria for irritable bowel syndrome: a critical review. Am J Gastroenterol 2010;105:814-820.

52. Agréus L, Svärdsudd K, Nyrén O, Tibblin G. Irritable bowel syndrome and dyspepsia in the general population: overlap and lack of stability over time. Gastroenterology 1995;109:671-680.

53. Jones R, Lydeard S. Irritable bowel syndrome in the general population. BMJ 1992;304:87-90.

54. Wilson S, Roberts L, Roalfe A, Bridge P, Singh S. Prevalence of irritable bowel syndrome: a community survey. $\mathrm{Br} \mathrm{J}$ Gen Pract 2004;54:495-502.

55. Bommelaer G, Poynard T, Le Pen C, et al. Prevalence of irritable bowel syndrome (IBS) and variability of diagnostic criteria. Gastroenterol Clin Biol 2004;28:554-561.

56. Dapoigny M, Bellanger J, Bonaz B, et al. Irritable bowel syndrome in France: a common, debilitating and costly disorder. Eur J Gastroenterol Hepatol 2004;16:995-1001.

57. Corazziari E, Attili AF, Angeletti C, De Santis A. Gallstones, cholecystectomy and irritable bowel syndrome (IBS) MICOL population-based study. Dig Liver Dis 2008;40:944-950.

58. Zagari RM, Law GR, Fuccio L, et al. Epidemiology of functional dyspepsia and subgroups in the Italian general population: an endoscopic study. Gastroenterology 2010;138:1302-1311.

59. Katsinelos P, Lazaraki G, Kountouras J, et al. Prevalence, bowel habit subtypes and medical care-seeking behaviour of patients with irritable bowel syndrome in Northern Greece. Eur J Gastroenterol Hepatol 2009;21:183-189.

60. Minocha A, Johnson WD, Abell TL, Wigington WC. Prevalence, sociodemography, and quality of life of older versus younger patients with irritable bowel syndrome: a population-based study. Dig Dis Sci 2006;51:446-453.

61. Choung RS, Locke GR, Schieck CD, Zinsmeister AR, Talley NJ. Do distinct dyspepsia subgroups exist in the community? A population-based study. Am J Gastroenterol 2007;102:1983-1989.

62. Jung HK, Choung RS, Locke GR 3rd, Schleck CD, Zinsmeister AR, Talley NJ. Diarrhea-predominant irritable bowel syndrome is associated with diverticular disease: a population-based study. Am J Gastroenterol 2010;105:652-661.

63. Thompson WG, Irvine EJ, Pare P, Ferrazzi S, Rance L. Functional gastrointestinal disorders in Canada: first population-based survey using Rome II criteria with suggestions for improving the questionnaire. Dig Dis Sci 2002;47:225-235.

64. Valerio-Ureña J, Vásquez-Fernández F, Jiménez-Pineda A, et al. Prevalence of irritable bowel syndrome in Veracruz City, Mexico: a community-based survey. Rev Gastroenterol Mex 2010;75:36-41.

65. Gómez Alvarez DF, Morales Vargas JG, Rojas Medina LM, et al. Prevalence of irritable bowel syndrome and associated factors according to the Rome III diagnostic criteria in a general population in Colombia. Gastroenterol Hepatol 2009;32:395-400.

66. Boyce PM, Talley NJ, Burke C, Koloski NA. Epidemiology of the functional gastrointestinal disorders diagnosed according to Rome II criteria: an Australian population-based study. Intern Med J 2006;36:28-36.

67. Massarrat S, Saberi-Firoozi M, Soleimani A, Himmelmann GW, Hitzges M, Keshavarz H. Peptic ulcer disease, irritable bowel syndrome and constipation in two populations in Iran. Eur J Gastroenterol Hepatol 1995;7:427-433.

68. Shah SS, Bhatia SJ, Mistry FP. Epidemiology of dyspepsia in the general population in Mumbai. Indian J Gastroenterol 2001;20: 103-106.

69. Ghoshal UC, Abraham P, Bhatt C, et al. Epidemiological and clin- 
ical profile of irritable bowel syndrome in India: report of the Indian Society of Gastroenterology Task Force. Indian J Gastroenterol 2008;27:22-28.

70. Ho KY, Kang JY, Seow A. Prevalence of gastrointestinal symptoms in a multiracial Asian population, with particular reference to reflux-type symptoms. Am J Gastroenterol 1998;93:1816-1822.

71. Chen LY, Ho KY, Phua KH. Normal bowel habits and prevalence of functional bowel disorders in Singaporean adults - findings from a community based study in Bishan. Community Medicine GI Study Group. Singapore Med J 2000;41:255-258.

72. Lau EM, Chan FK, Zie ET, Chan CS, Wu JC, Sung JJ. Epidemiology of irritable bowel syndrome in Chinese. Dig Dis Sci 2002;47:2621-2624.

73. Zuckerman MJ, Nguyen G, Ho H, Nguyen L, Gregory GG. A survey of irritable bowel syndrome in Vietnam using the Rome criteria. Dig Dis Sci 2006;51:946-951.

74. Cheung TK, Lam KF, Hu WH, et al. Positive correlation between gastro-oesophageal reflux disease and irritable bowel syndrome in a Chinese population. Aliment Pharmacol Ther 2007;25:1099-1104.

75. Lu CL, Chen CY, Lang HC, et al. Current patterns of irritable bowel syndrome in Taiwan: the Rome II questionnaire on a Chinese population. Aliment Pharmacol Ther 2003;18:1159-1169.

76. Kawamura A, Adachi K, Takashima T, Yuki M, Ono M, Kinoshita Y. Prevalence of irritable bowel syndrome and its relationship with Helicobatcer pylori infection in a Japanes population. Am J Gastroenterol 2001;96:1946.

77. Yilmaz S, Dursun M, Ertem M, Canoruc F, Turhanoğlu A. The epidemiological aspects of irritable bowel syndrome in Southeastern Anatolia: a stratified randomized community-based study. Int J Clin Pract 2005;59:361-369.

78. Basaranoglu M, Celebi S, Ataseven H, Rahman S, Deveci SE, Acik Y. Prevalence and consultation behavior of self-reported rectal bleeding by face-to-face interview in an Asian community. Digestion 2008;77:10-15.

79. Hoseini-Asi MK, Amra B. Prevalence of irritable bowel syndrome in Shahrekord, Iran. Indian J Gastroenterol 2003;22:215-216.

80. Sohrabi S, Nouraie M, Khademi H, Baghizadeh S, Nasseri-Moghaddam S, Malekzadeh R. Epidemiology of uninvestigated gastrointestinal symptoms in adolescents: a population-based study applying the Rome II questionnaire. J Pediatr Gastroenterol Nutr 2010; 51:41-45.

81. Yarandi SS, Nasseri-Moghaddam S, Mostajabi P, Malekzadeh R. Overlapping gastroesophageal reflux disease and irritable bowel syndrome: increased dysfunctional symptoms. World J Gastroenterol 2010;16:1232-1238.

82. Jafri W, Yakoob J, Jafri N, Islam M, Ali QM. Irritable bowel syndrome and health seeking behaviour in different community of Pakistan. J Pak Med Assoc 2007;57:285-287.

83. Masud MA, Hasan M, Khan AK. Irritable bowel syndrome in a rural community in Bangladesh: prevalene, symptoms pattern, and health care seeking behavior. Am J Gastroenterol 2001;96:15471552.

84. Perveen I, Hasan M, Masud MA, Bhuiyan MM, Rahman MM. Irritable bowel syndrome in a Bangladeshi urban community: prevalence and health care seeking pattern. Saudi J Gastroenterol 2009; 15:239-243.
85. Devanarayana NM, Adhikari C, Pannala W, Rajindrajith S. Prevalence of Functional Gastrointestinal Diseases in a Cohort of Sri Lankan Adolescents: Comparison Between Rome II and Rome III Criteria. J Trop Pediatr Published Online First: 4 Jun 2010. doi:10.1093/tropej/fmq039

86. Rajendra S, Alahuddin S. Prevalence of irritable bowel syndrome in a multi-ethnic Asian population. Aliment Pharmacol Ther 2004; 19:704-706.

87. Kwan AC, Hu WH, Chan YK, Yeung YW, Lai TS, Yuen H. Prevalence of irritable bowel syndrome in Hong Kong. J Gastroenterol Hepatol 2002;17:1180-1186.

88. Lee S, Wu J, Ma YL, Tsang A, Guo WJ, Sung J. Irritable bowel syndrome is strongly associated with generalized anxiety disorder: a community study. Aliment Pharmacol Ther 2009;30:643-651.

89. Wang AJ, Liao XH, Hu PJ, Liu SC, Xiong LS, Chen MH. A comparison between Rome III and II criteria in diagnosing irritable bowel syndrome. Zhonghua Nei Ke Za Zhi 2007;46:644-647.

90. Dai N, Cong Y, Yuan H. Prevalence of irritable bowel syndrome among undergraduates in Southeast China. Dig Liver Dis 2008;40: 418-424.

91. Shen L, Kong H, Hou X. Prevalence of irritable bowel syndrome and its relationship with psychological stress status in Chinese university students. J Gastroenterol Hepatol 2009;24:1885-1890.

92. Zhao Y, Zou D, Wang R, et al. Dyspepsia and irritable bowel syndrome in China: a population-based endoscopy study of prevalence and impact. Aliment Pharmacol Ther Published Online First: 22 May 2010. doi: 10.1111/j.1365-2036.2010.04376.x

93. Lee SY, Lee KJ, Kim SJ, Cho SW. Prevalence and risk factors for overlaps between gastroesophageal reflux disease, dyspepsia, and irritable bowel syndrome: a population-based study. Digestion 2009; 79:196-201.

94. Son YJ, Jun EY, Park JH. Prevalence and risk factors of irritable bowel syndrome in Korean adolescent girls: a school-based study. Int J Nurs Stud 2009;46:76-84.

95. Park DW, Lee OY, Shim SG, et al. The differences in prevalence and sociodemographic characteristics of irritable bowel syndrome according to Rome II and Rome III. J Neurogastroenterol Motil 2010;16:186-193.

96. Shiotani A, Miyanish T, Takahashi T. Sex differences in irritable bowel syndrome in Japanese university students. J Gastroenterol 2006;41:562-568.

97. Shinozaki M, Fukudo S, Hongo M, et al. High prevalences of irritable bowel syndrome in medical outpatients in Japan. J Clin Gastroenterol 2008;42:1010-1016.

98. Hori K, Matsumoto T, Miwa H. Analysis of the gastrointestinal symptoms of uninvestigated dyspepsia and irritable bowel syndrome. Gut Liver 2009;3:192-196.

99. Okumura T, Tanno S, Ohhira M, Tanno S. Prevalence of functional dyspepsia in an outpatient clinic with primary care physicians in Japan. J Gastroenterol 2010;45:187-194.

100. Kaji M, Fujiwara Y, Shiba M, et al. Prevalence of overlaps between GERD, FD and IBS and impact on health-related quality of life. J Gastroenterol Hepatol 2010;25:1151-1156.

101. Khoshkrood-Mansoori B, Pourhoseingholi MA, Safaee A, et al. Irritable bowel syndrome: a population based study. J Gastrointestin Liver Dis 2009;18:413-418. 
102. Wang A, Liao X, Xiong L, et al. The clinical overlap between functional dyspepsia and irritable bowel syndrome based on Rome III criteria. BMC Gastroenterol 2008;8:43-49.

103. Nam SY, Kim BC, Ryu KH, Park BJ. Prevalence and risk factors of irritable bowel syndrome in healthy screenee undergoing colonoscopy and laboratory tests. J Neurogastroenterol Motil 2010;16: 47-51.

104. Noh YW, Jung HK, Kim SE, Jung SA. Overlap of erosive and non-erosive reflux diseases with functional gastrointestinal disorders according to Rome III criteria. J Neurogastroenterol Motil 2010; 16:148-156.

105. Chang FY, Lu CL. Irritable bowel syndrome in the 21 st century: perspectives from Asia or Southeast Asia. J Gastroenterol Hepatol 2007;22:4-12

106. Gwee KA, Bak YT, Ghoshal UC, et al. Asian consensus on irritable bowel syndrome. J Gastroenterol Hepatol 2010;25:1189-1205.

107. Faresjö A, Grodzinsky E, Johansson S, Wallander MA, Timpka T, Akerlind I. A population-based case-control study of work and psychosocial problems in patients with irritable bowel syndrome, women are more seriously affected than men. Am J Gastroenterol 2007;102:371-379.

108. Hammer J, Talley NJ. Value of different diagnostic criteria for the irritable bowel syndrome among men and women. J Clin Gastroenterol 2008;42:160-166.

109. Lu CL, Chang FY, Lang HC, Chen CY, Luo JC, Lee SD. Gender difference on the symptoms, health seeking behavior, social impact and sleep quality in irritable bowel syndrome: a Rome II-based survey in an apparent healthy adult Chinese population in Taiwan. Aliment Pharmacol Ther 2005;21:1497-1505.

110. Si JM, Wang LJ, Chen SJ, Sun LM, Dai N. Irritable bowel syndrome consulters in Zhejiang province: the symptoms pattern, predominant bowel habit subgroups and quality of life. World $\mathrm{J}$ Gastroenterol 2004;10:1059-1064.

111. Fang X, Lu S, Pan G. An epidemiologic study of bowel habit in adult non-patient population in Beijing. Zhonghua Yi Xue Za Zhi 2001;81:1287-1290.

112. Rubin G, De Wit N, Meineche-Schmidt V, Seifert B, Hall N, Hungin $\mathrm{P}$. The diagnosis of IBS in primary care: consensus development using nominal group technique. Fam Pract 2006;23: 687-692.

113. Schmulson M, Adeyemo M, Gutiérrez-Reyes G, et al. Differences in gastrointestinal symptoms according to gender in Rome II positive IBS and dyspepsia in a Latin American population. Am J Gastroenterol 2010;105:925-932. 